\title{
Adhesion and neurite development of cortical neurons on micropatterns of polyethylenimine and fluorocarbon
}

\author{
T.G. Ruardij (1), M.H. Goedbloed (1), and W.L.C. Rutten (1) \\ (1) Institute for Biomedical Technology, Faculty of Electrical Engineering, University of Twente, P.O. Box 217, 7500 AE \\ Enschede, The Netherlands, T.G.Ruardij@el.utwente.nl,
}

\begin{abstract}
This study aims on the preparation of isolated islands of cortical neurons on modified glass surfaces. Isolated islands of cortical neurons were obtained with a combination of neuron-adhesive polyethylenimine (PEI) and neuron-repellent plasma-deposited fluorocarbon (FC). Neurite development and fasciculation of interconnecting neurites between PEI-coated areas was studied. The patterns consisted of PEI-coated wells (diameter $150 \mu \mathrm{m}$ ) which were initially etched in a Fluorocarbon (FC) layer. The separation distance between the PEI-coated wells was varied from 10 up to $90 \mu \mathrm{m}$. This paper shows that the chemical patterns, prepared with a combination of polyethylenimine (PEI) and plasma deposited Fluorocarbon (FC), results in highly compliant patterns of adhering cortical neurons. Furthermore, it was shown that the occurrence of connecting neurite fascicles between neurons on PEI-coated wells is inversely proportional to the separation distance between the wells. Interconnecting fascicles were especially present on the pattern with a minimal separation distance $(10 \mu \mathrm{m})$ between the PEI-coated wells. In contrast, interconnecting neurite fascicles were not observed on patterns with a minimal separation distance of $80 \mu \mathrm{m}$ between the wells.
\end{abstract}

Key words - Cortical neurons, Polyethylenimine, Fluol:ocarbon

\section{INTRODUCTION}

Maintenance of patterned neurons on planar surfaces is most likely to be achieved with a stable and chemically inert combination of adhesive and non-adhesive materials. Coatings of biomolecules [1] or frequently used chemical coatings $[2,3]$ do not fully meet these necessary requirements to survive under physiological circumstances over longer periods of time.

Many authors stressed the important role of Fluorocarbon (FC) coatings as tools to prepare non-adhesive surfaces for cells [4,5]. The properties of FC-coatings resemble the properties of Polytetrafluoroethylene (PTFE), a material with exceptional thermal, electrical and chemical properties [6]. The strong irreversible adsorption of albumin on hydrophobic PTFE is considered to be the important factor in preventing specific binding between cells and PTFE $[1,6]$. The properties of PTFE are considered to be indicative for a whole range of FC-coatings. Some techniques that are available to deposit FC-coatings on surfaces are spincoatings of commercially available products, plasma de- posited coatings of carbonhydrotrifluoride $\left(\mathrm{CHF}_{3}\right)$ and $\mathrm{E}$ beam evaporated coatings of PTFE [7].

Cell adhesive coatings with the ability to maintain their surface properties in a physiological environment are rare. Examples of disturbing processes are the desorption of the top layer of self assembled monolayers of thiols on gold surfaces [8] or the hydrolysis of silanes [9] and polypep. tides in other studies. The application of these coatings is therefore more questionable for long-term patterning of cells. However the choice for many of these coatings is driven by the supposed electrostatic interaction between positively charged aminogroups and negatively charged phospholipids in the cell membrane [10]. A clear example is poly-L-lysine with a high density of positively charged amino groups in the peptide chain [11]. An interesting alternative and relatively unknown material in the field of neuroscience is polyethylenimine [12]. Polyethylenimine (PEI) in aqueous solution contains a relatively high density of positively charged aminogroups in the polymer structure and is expected to be more bioresistant due to the lack of amide linkages in the polymer backbone. PEI behaves like a positively charged polyelectrolyte in an aqueous solution below $\mathrm{pH} 9$ and fixates electrostatically onto negatively charged materials like mica or silicondioxide $[10,12]$. The unique electrostatic properties of PEI offer the possibility to form a stable binding between neuron membranes and inert negatively charged surfaces $[10,12]$.

The aim of this paper is to prepare isolated islands of cortical neurons on glass surfaces modified with a combination of neuron-adhesive polyethylenimine (PEI) and a neuron-repellent plasma-deposited fluorocarbon (FC) layer. Adhesion and development (fasciculation) of neurites between PEI-coated areas, embedded in the plasma-deposited FC layer is studied. In addition, viability of neurons was assessed over a time period of 8 days.

\section{MATERIALS AND METHODS}

Glass (N.V.Glaverbel, Mol, Belgium) was used as a base material onto which different coatings were prepared. Initially glass samples were spin coated with polyimide (Olin microelectronic materials) which was diluted in n-methyl pyrolidon (1:1 volume ratio). Samples were post-baked at $120^{\circ} \mathrm{C}$ during 5 minutes to remove residual solvent. The polyimide (PI) samples were used for the deposition of neurophilic and neurophobic coatings.

Neurophilic coating and deposition

$1^{\text {st }}$ Annual International IEEE-EMBS Special Topic Conference on Microtechnologies in Medicine \& Biology 
Polyethyleneimine (PEI):

A stock solution $(10 \mathrm{mg} / \mathrm{ml})$ of Polyethylenimine in water (Fluka chemie, MW between $6 * 10^{5}$ and $1 * 10^{6}$ ) was diluted 1000 times to reach a final concentration of $10 \mu \mathrm{g} / \mathrm{ml}$. Under sterile conditions each sample was immersed into this solution during a time period of 60 seconds. Each sample was rinsed extensively in sterile millipore water to remove some non-adsorbed PEI.

\section{Neurophobic coating and deposition}

Plasma deposited Fluorocarbon (FC):

Samples were coated with a $\mathrm{FC}$-layer using a $\mathrm{CHF}_{3}$ plasma (25 sccm, $150 \mathrm{mT}$ Trr, $10 \mathrm{~W}$ ) for 9.5 minutes and a subsequent treatment with $\mathrm{CHF}_{3}(5 \mathrm{~W})$ for $1.5 \mathrm{~min}$.

\section{Fabrication procedures of patterned surfaces}

In a first step the FC-layer is deposited onto the Polyimide coated glass. In a second step a pattern of wells (diameter of $150 \mu \mathrm{m}$ ) was etched in the combined polyimide/fluorocarbon layer using microlithography [3]. The pattern consisted of 9 different subsections with each subsection having a different separation distance between the wells (Fig. 1). The distance between the circular PEl-coated wells was varied between 10 and $90 \mu \mathrm{m}$. Selective removal of the combined polyimide/ fluorocarbon layer was achieved with a $\mathrm{CHF}_{3} / \mathrm{O}_{2}$ plasma $\left(25 \mathrm{sccm} \mathrm{CHF}_{3}, 5 \mathrm{sccm}\right.$ $\mathrm{O}_{2}, 10 \mathrm{mT}$ orr and $75 \mathrm{~W}$ ). Subsequently the samples were immersed in the Polyethylenimine (PEI) solution for 60 seconds. Finally the protective photoresist on top of the fluorocarbon layer was lifted off with a $\mathrm{NaOH}$ solution (1.0 M) and simultaneously lifted off PEI from the areas around the wells (Fig.2).

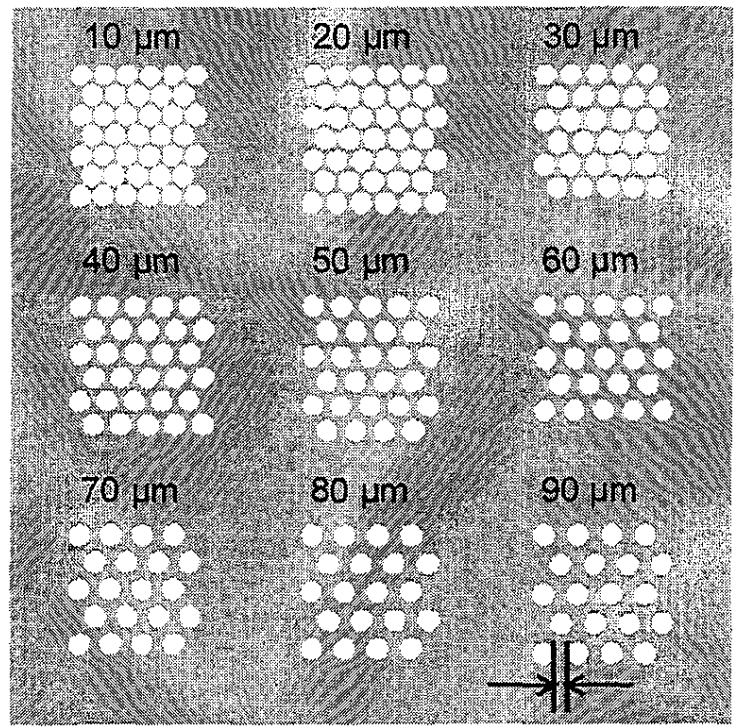

Fig.1. Overview of the patterns of wells, used for the experiments. Dark areas represent the Fluorocarbon (FC) coated part of surface. Light areas represent the Polyethylenimine (PEI) coated part of the surface. The numbers refer to the separation distance between the PEI-coated wells. Diameter of wells is $150 \mu \mathrm{m}$.

\section{PEl lift-off method}

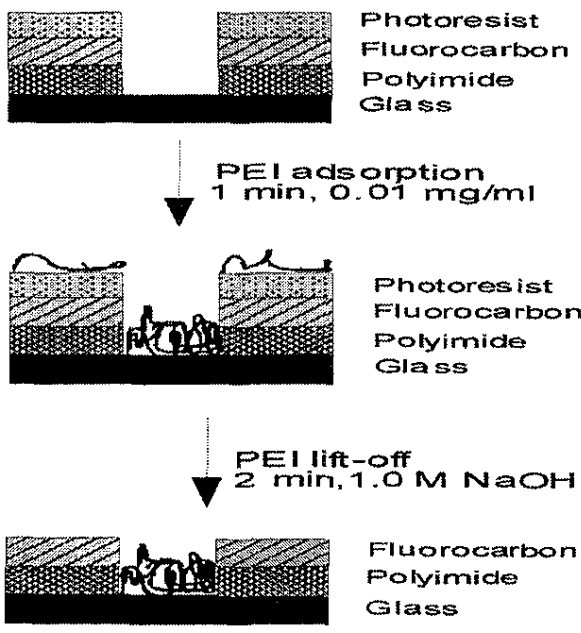

Fig.2. Procedure to prepare chemical micropatterns with Polycthylenimine (PEI) and Fluorocarbon (FC).

\section{Cortical neuron isolation}

Cerebral cortex from 1 day old newborn rats was dissected out under sterile circumstances. The tissue was trypsinized ( $0.25 \%$ Trypsin/EDTA) for 45 minutes in an incubator at $37^{\circ} \mathrm{C}$ at $5 \% \mathrm{CO}_{2}$ and subsequently treated with Soybean Trypsin Inhibitor (STl, $1 \mathrm{mg} / \mathrm{ml}$ ) and Desoxyribonuclease I (DNAse I, 1.1 unit/ml). Dissociated tissue was spun down at $1200 \mathrm{rpm}$ during 5 minutes and resuspended in R12 medium. Sedimentation of cortical neurons onto patterned surfaces took place from a glass cylinder with a sedimentation depth of $5 \mathrm{~mm}$. The applied seeding density of cortical neurons was $0.15^{*} 10^{6}$ cells $/ \mathrm{ml}$. After 4 hours of adhesion, samples were rinsed with a physiological salt solution $(0.9$ $\% \mathrm{NaCl}$ ) to remove non-adherent neurons.

\section{Neuron viability staining}

Over a time period of 8 days, viability of neurons was evaluated using a staining procedure with Propidium Iodide (PI, $5 \mu \mathrm{g} / \mathrm{ml}$ ) and Acridine Orange (AO, $1.5 \mu \mathrm{g} / \mathrm{ml}$ ). Chemicals were purchased from Sigma Aldrich (Zwijndrecht, The Netherlands) and diluted in Phosphate Buffered Saline. AO-PI stained neurons were examined with epi-fluorescence equipment, mounted on an Nikon inverted microscope Diaphot-TMD (Nikon, Japan). The 450$490 \mathrm{~nm}$ excitation filter in combination with a $510 \mathrm{~nm}$ dichroic mirror and $520 \mathrm{~nm}$ barrier filter permitted the simultaneous observation of both viable (green) and nonviable (red) neurons. Photomicrographs were taken with a Nikon-FE $35 \mathrm{~mm}$ camera, attached to the front camera port. Photomicrographs were processed in Microsoft compatible software (Coral PhotoPaint 7, Corel Corporation, Ontario, Canada) to determine the fraction of the surface covered with viable (green) and non-viable (red) neurons. Viability of cortical neurons was evaluated on the $90 \mu \mathrm{m}$ spaced pattern (see Fig.1) after 1, 4, and 8 days.

$6361^{\text {st }}$ Annual International IEEE-EMBS Special Topic Conference on Microtechnologies in Medicine \& Biology October 12-14, 2000, Lyon, France - 0-7803-6603-4/00/\$10.0002000 IEEE 


\section{RESULTS AND DISCUSSION}

The adhesion of cortical neurons on the patterned surfaces was investigated. Two examples of cortical neuron adhesion after 1 day in vitro are presented in Fig.3. The results emphasize some typical phenomena observed during the experiments. Polyethylenimine (PEI) was an excellent neurophilic coating and supported the adhesion of cortical neurons inside the PEI-coated wells to a significant extent. Statistical analysis revealed that $85 \pm 10 \%$ of the total number of observed neurons were located inside the PEIcoated wells on patterned surfaces with only $35 \%$ covered with PEI-coated circular wells (Fig.3, right side). The $\mathrm{CHF}_{3}$ plasma deposited Fluorocarbon layer served as a nonadhesive coating for cortical neurons (see Fig.3).
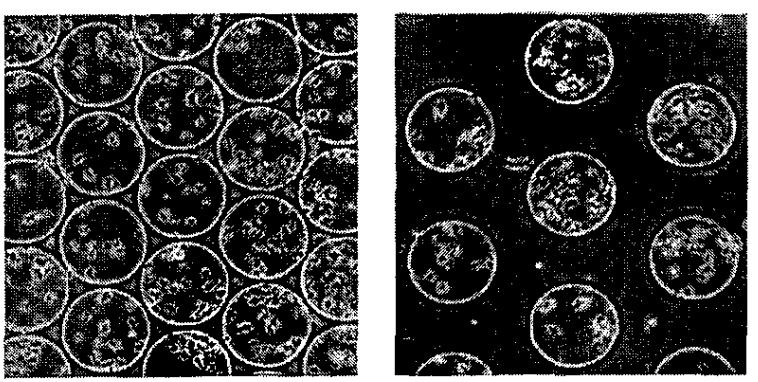

Fig.3. Phase contrast images of cortical neuron distribution on patterned surfaces after 1 day in vitro. Left: cortical neuron distribution on a pattern with a separation distance of $10 \mu \mathrm{m}$ between the PEI-coated wells. Right: contical neuron distribution on a pattern with a separation distance of 90 $\mu \mathrm{m}$

Fig.4 shows morphological features of the developing cortical neurons after 8 days in vitro on the same sections as shown in Fig.3. A clear clustering of neurons into aggregates is visible on both patterns. The center of neuron aggregates is frequently positioned inside the well if the
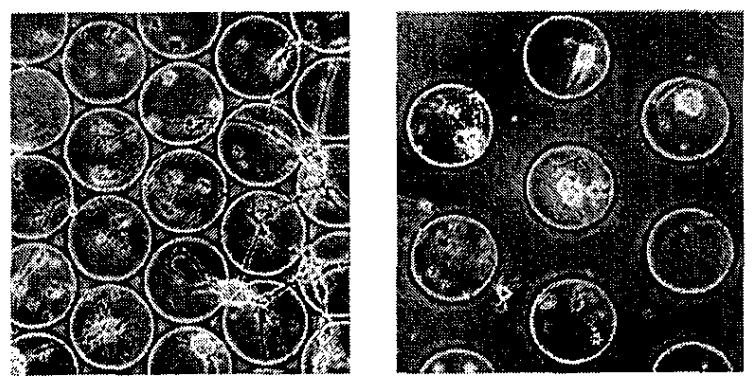

Fig.4. Examples of cortical neuron aggregation and development of interm connecting neurite fascicles between PEI-coated wells after 8 days in vitro on patterned surfaces with typical separation distances of $10 \mu \mathrm{m}$ (left) and 90 um (right) between the PEI-coated weils.

wells are more separated from each other (Fig.3, right side). On the pattern with small distances between the wells, aggregates can also be found in between. Fig.5 shows the corresponding quantitative result for the 9 different patterns. Due to the structure of the patterns, each PEI-coated well is surrounded by 6 other wells. Thus the number of interconnecting neurite fascicles between a neurophilic well and its surrounding wells is always limited to a maximum number of 6 . Fig. 5 demonstrates that interconnecting neurite

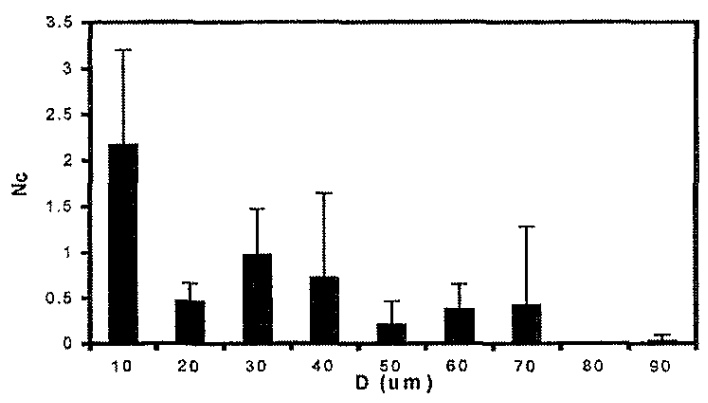

Fig.5. Average number $\mathrm{Nc}$ of observed interconnecting neurite fascicles between a PEl-coated well and the 6 surrounding wells vs. the separation distance D between the PEl-coated wells. Mean \pm S.D. (calculated over 5 images).

fascicles between neurophilic PEI-coated wells were especially present on the pattern with a minimal distance of 10 $\mu \mathrm{m}$ between the neurophilic PEI-coated wells (Fig.4, left side). In this case, the average number of connections still was only 2.2 and did not approach the maximal number of 6. The conclusion is that the $\mathrm{CHF}_{3}$ plasma deposited fluorocarbon layer is a non-adhesive surface for cortical neurons and additionally serves as a barrier for developing neurites. Complete isolation of neurons and neurites into neurophilic PEI-coated wells was obtained with a minimal separation distance of $80 \mu \mathrm{m}$ in between (Fig.5).

Fig. 5 shows that a distinct part of the adhering neurons lost their viability on the PEI-coated wells in a time period of 8 days. The general observation is that a large number of neurons form aggregates after active migration of the adhering cells towards each other. The data suggests that neurons within an aggregate lose their viability through a lack of nutrition, which is caused by a limited diffusion of medium components towards the encapsulated neurons. An important observation, supporting this hypothesis, is the fact that areas with yellow tissue could clearly be identified, apart from the expected red (PI-stained) and green ( $\mathrm{AO}$ stained) tissue (Fig.6). The yellow color, a result of this

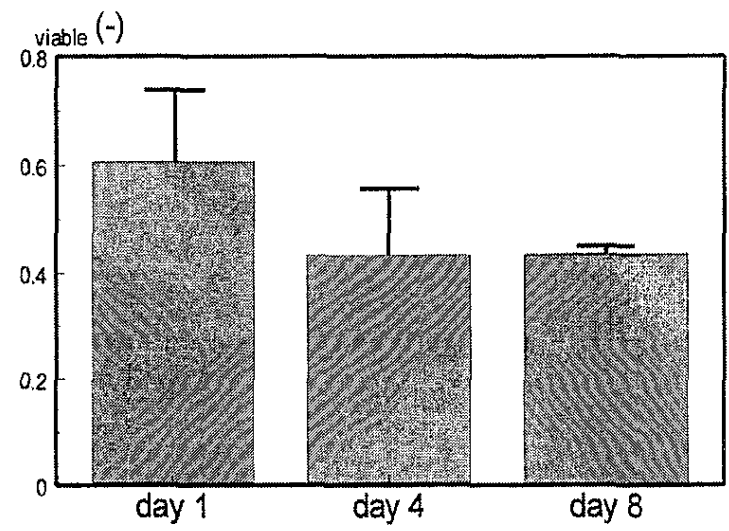

Fig.5. Fractional surface coverage of PEl-coated wells with viable neurons $\mathrm{F}$ viable after 1,4 and 8 days. Mean $=$ S.D. (calculated over $4 \mathrm{im}$ ages).

$1^{\text {st }}$ Annual International IEEE-EMBS Special Topic Conference on Microtechnologies in Medicine \& Biology 


\section{REFERENCES}
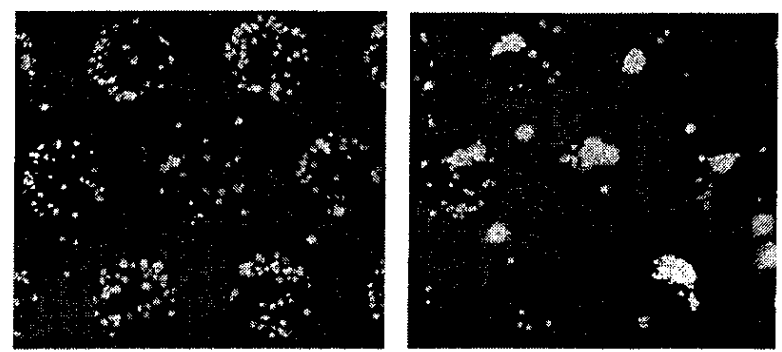

Fig.6. Fluorescent images of Acridine Orange (green)-Propidium lodide (red) stained cortical neurons after 1 day (left) and 8 days (right). Results are shown on a $90 \mu \mathrm{m}$ spaced pattern. Dark areas represent the PEI-coated wells.

staining procedure, can be explained as the cumulative fluorescence of red (non-viable) and green (viable) neurons overlapping each other [13]. The yellow color, especially observed in neuronal aggregates, suggests the presence of both living and dead neurons overlapping each other.

\section{CONCLUSION}

The plasma-deposited Fluorocarbon ( $\mathrm{FC}$ ) layer is a nonadhesive coating for cortical neurons as opposed to Polyethylenimine (PEI) which supports neuronal adhesion extensively. Development of fasciculated neurites between PEI-coated wells is inversely proportional to the separation distance in between. Over a time period of 8 days, complete isolation of neurons and neurites is found on $80 \mu \mathrm{m}$ spaced patterns. The fraction of the surface, covered with viable tissue, is decreasing from 0.6 down to 0.4 as was determined with a double staining procedure using propidium iodide and acridine orange. After staining, the color of aggregates is predominantly yellow, which is the expected cumulative effect of green (living) and red (dead) neurons overlapping each other [13].
[1] K. Lewandowska, E. Pergament, C.N. Sukenik, L.A. Culp, Cell-type specific adhesion mechanisms mediated by fibronectin adsorbed to chemically derivatized substrata, $J$. Biomed. Mater. Res., 26, $1343-$ 1363 (1992)

[2] D. Kleinfeld, K.H. Kahler, and P.E. Hockberger, "Controlled outgrowth of dissociated neurons on patterned substrates", $J$. of Neuroscience, 8, 4098-4120 (1988).

[3] J.M. Corey, B.C. Wheeler, and G.J. Brewer, "Micrometerresolution silane-based patterning of hippocampal neurons: critical variables in photoresist and laser ablation processes for substrate fabrication", IEEE Trans. Biomed. Eng, 43, 944-955 (1996).

[4] J.P. Ranieri. R. Bellamkonda, E.J. Bekos, T.G. Vargo, J.A. Gardella, $\mathrm{Jr}_{\text {. P. Aebisher, "Neuronal cell attachment to fluorinated ethylene }}$ propylene films with covalently immobilized oligopeptides YIGSR and IVKAV. II", J. Biomed. Mater. Res., 29, 779-785, 1995.

[5] S.A. Makohliso, L. Giovangrandi, D. Leonard, H.J. Mathieu, M. llegems, and P. Aebisher, "Application of Teflon-AF@ thin films for bio-patterning of neural cell adhesion", Biosensors \& Bioelectronics, $13,1227-1235$ (1998).

[6] C.J. van Oss, Interfacial forces in aqueous media, Marcel Dekker Inc, New York, pp336-352 (1994).

[7] H.V. Jansen, J.G.E. Gardeniers, J. Elders, H.A.C. Tilmans and M. Elwenspoek, "Applications of fluorocarbon polymers in micromechanics and micromachining", Sensors and Actuators A, 41-42, 136140 (1994).

[8] C.A. Scotchford, E. Cooper, G.J. Legget, S. Downes, "Growth of human osteoblast-Iike cells on alkanethiol on gold self assembled monolayers"; the effect of surface chemistry", J. Biomed. Mater. Res. 41, 431-442 (1998)

[9] S.W. Klintstrom, Ellipsometry and wettability surfaces, PhD thesis, Linkoping university, Sweden, pp 10-17 (1992).

[10]J.Y. Wong, C.K. Park, M. Seitz, and J. Israelachvili, "Polymercushioned bilayers. II. An investigation of interaction forces and fusion using the surface forces apparatus" Biophys. $J$., 77, [458-1468 (1999).

[11]D.A.Stenger, T.M. McKenna (eds.), Enabling technologies for cultured neural networks, Academic Press, London, 1994.

[12] T.P. Golub, A.L Skachkova, M.P. Sidorova, Investigation of surface reactions on an interface of $\mathrm{SiO}_{2}$ with an aqueous solution of polyethylenimine. Colloid Journat of the Russian Accademy of Sciences, $54,697.702$ (1991).

[13] H.L.Bank, "Rapid assessment of islet viability with acridine orange and propidium iodide", In vitro Cell. Dev. Biol., 24, 266-273 (1988). 